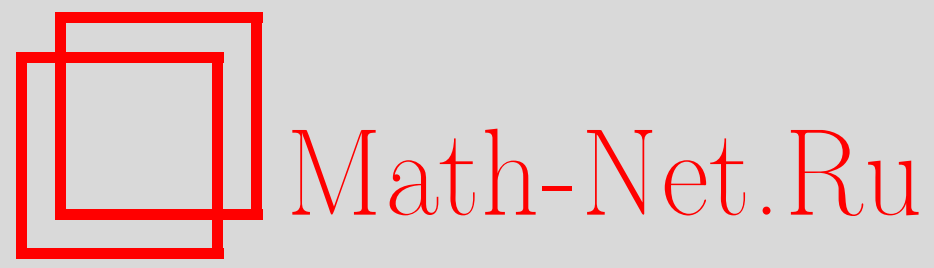

В. Г. Михайлов, А. М. Шойтов, О числах множеств эквивалентных цепочек в последовательности независимых случайных величин, Матем. вопр. криптогр., 2013, том 4, выпуск 1, 77-86

DOI: https://doi.org/10.4213/mvk74

Использование Общероссийского математического портала Math-Net.Ru подразумевает, что вы прочитали и согласны с пользовательским соглашением

http://www.mathnet.ru/rus/agreement

Параметры загрузки:

IP: 3.89 .197 .203

26 апреля 2023 г., 14:16:56 
УДК: 519.212.2+519.214.5

\title{
О числах множеств эквивалентных цепочек \\ в последовательности независимых случайных величин
}

\author{
В. Г. Михайлов ${ }^{1}$, А. М. Шойтов ${ }^{2}$ \\ ${ }^{1}$ Математический институт им. В. А. Стеклова РАН, Москва \\ ${ }^{2}$ Академия криптографии Российской Федерации, Москва
}

Получено 3.Х.2012

В работе выведены предельные теоремы для распределения вектора из чисел $r$-множеств эквивалентных $s$-цепочек в последовательности $\mathbf{X}$ из $n+s-1$ независимых одинаково распределенных на множестве $\{1, \ldots, N\}$ случайных величин. Рассматриваются две области изменения параметров при $n, N \rightarrow \infty, s<N$. В первой из них при переходе к пределу ограничено среднее число $s$-цепочек из элементов $\mathbf{X}$, имеющих совпадения знаков в своей записи, а во второй, напротив, ограничено среднее число $s$-цепочек, которые не имеют совпадений знаков.

Ключевые слова: полиномиальные испытания, эквивалентные цепочки, сложное пуассоновское распределение

On the numbers of equivalent tuples sets in a sequence of independent random variables

\author{
V. G. Mihailov ${ }^{1}$, A. M. Shoitov ${ }^{2}$ \\ ${ }^{1}$ Steklov Mathematical Institute of RAS, Moscow \\ ${ }^{2}$ Academy of Cryptography of the Russian Federation, Moscow
}

\begin{abstract}
Let $\mathbf{X}$ be a sequence of $n+s-1$ polynomial trials with $N$ outcomes. Limit joint distributions of the numbers of $r$-sets of equivalent $s$-tuples in $\mathbf{X}$ are proved. Two types of conditions on the parameters $n, N \rightarrow \infty, s<N$ are considered. Under the conditions of the first type the mean number of $s$-tuples with coinciding outcomes is bounded. Under the conditions of the second type the mean number of $s$-tuples without concidings is bounded.
\end{abstract}

Key words: polynomial trials, equivalent tuples, compound Poisson distribution

Citation: Mathematical Aspects of Cryptography, 2013, vol. 4, no. 1, pp. 77-86 (Russian). 


\section{1. Введение}

Задача об эквивалентных цепочках в случайных дискретных последовательностях ведет свое начало с работы [1]. Напомним, что цепочки $\left(x_{1}, \ldots, x_{s}\right)$ и $\left(y_{1}, \ldots, y_{s}\right)$ называют эквивалентными, если существует такая перестановка $\sigma=(\sigma(1) \ldots, \sigma(N))$ знаков алфавита $\{1, \ldots, N\}$, при которой $\left(y_{1}, \ldots, y_{s}\right)=\left(\sigma\left(x_{1}\right), \ldots, \sigma\left(x_{s}\right)\right)$. Эквивалентные цепочки всегда имеют одинаковую структуру, а именно, если $x_{i}=x_{j}$, то и $y_{i}=y_{j}$, и наоборот (подробности см. в [2]). Свойство эквивалентности цепочек $\left(x_{1}, \ldots, x_{s}\right)$ и $\left(y_{1}, \ldots, y_{s}\right)$ будем обозначать $\left(x_{1}, \ldots, x_{s}\right) \sim\left(y_{1}, \ldots, y_{s}\right)$. Структуру цепочки, все знаки которой различны, будем называть тривиальной. Все такие цепочки эквивалентны между собой.

Пусть $\mathbf{X}=\left(X_{1}, X_{2}, \ldots\right)$ - последовательность независимых случайных величин, принимающих значения в множестве $\{1, \ldots, N\}$ с вероятностями $\mathbf{P}\left\{X_{t}=k\right\}=p_{k}, k=1, \ldots, N$. Положим $P_{r}=\sum_{k=1}^{N} p_{k}^{r}$.

Зададим натуральные числа $s, n, s<n$, и рассмотрим последовательность

$$
\mathbf{Y}(n, s)=\left\{Y_{1}(s), \ldots, Y_{n}(s)\right\}, \quad Y_{t}(s)=\left(X_{t}, \ldots, Y_{t+s-1}\right),
$$

$s$-цепочек, встретившихся на начальном отрезке (длины $n+s-1$ ) последовательности $\mathbf{X}$. Введем случайные величины: $\mu_{r}=\mu_{r}(N, n, s)-$ число множеств попарно эквивалентных цепочек в $\mathbf{Y}(n, s)$, состоящих из $r \geq 2$ цепочек, $\mu_{1}=\mu_{1}(N, n, s)$ - число цепочек в $\mathbf{Y}(n, s)$, не имеющих в этой последовательности себе эквивалентных, и $\mu=\sum_{r \geq 1} \mu_{r}(N, n, s)$ - число всех таких множеств.

Если интерпретировать множество всех возможных структур $s$-цепочек как множество ячеек, а появление в $\mathbf{Y}(n, s) s$-цепочки с определенной структурой - как помещение частицы в ячейку, отвечающую этой структуре, мы получим схему зависимого размещения частиц, в которой величины $\mu_{r}=\mu_{r}(N, n, s)$ имеют традиционный смысл: это числа ячеек, в каждую из которых попало $r$ частиц, $r=0,1, \ldots$, а $\mu=\mu(N, n, s)$ - число занятых ячеек.

В настоящей работе исследуется предельное распределение вектоpa $M_{R}=\left(\mu_{1}, \mu_{2}, \ldots, \mu_{R}\right)$ (здесь $R-$ натуральное число) в случае, когда $n, N \rightarrow \infty$, а параметр $s$ остается постоянным или растет вместе с $n, N$ согласованным с ними образом, но $s<N$.

В разделе 2 рассматривается последовательность исходов полиномиальной схемы, причем параметры $n, N \rightarrow \infty$ и $p_{1}, \ldots, p_{N}$ при переходе к пределу меняются так, что ограничено среднее число $s$-цепочек элементов рассматриваемой последовательности, имеющих совпадения знаков в своей за- 
писи. В этом случае свойства вектора $M_{R}$ определяются цепочками, имеющими лишь одно совпадение знаков. Такую структуру цепочки будем называть простейшей структурой. Здесь выводятся оценки скорости сближения распределения вектора $M_{R}$ с сопровождающим дискретным распределением и выводится соответствующая предельная теорема для случая $s=$ const $\geq 2$. Показано также, что в этом случае при $s \rightarrow \infty$ для величин $\mu_{r}$ возможны только несобственные либо сосредоточенные в нуле дискретные предельные распределения.

В разделе 3 рассматривается случай, когда $p_{k}=1 / N, k=1, \ldots, N$, $n, N \rightarrow \infty, s N^{-1} \rightarrow \lambda \in(0,1)$. Накладывается также условие, ограничивающее среднее число $s$-цепочек, которые не имеют в своей записи совпадений знаков. Доказано, что в этом случае распределение бесконечномерного вектора $\left(\mu_{2}, \mu_{3}, \ldots\right)$ сходится к специальному бесконечномерному дискретному распределению.

\section{2. Случай, когда среднее число $s$-цепочек с нетривиальной структурой ограничено}

В этом разделе рассмотрим случай, когда в последовательности $\mathbf{Y}(n, s)$ имеются главным образом цепочки с не более чем одним повторением символов (цепочки с тривиальной и простейшей структурами). Изучается распределение вектора

$$
M_{R}=\left(\mu_{1}, \mu_{2}, \ldots, \mu_{R}\right), \quad R \geq 1 .
$$

Рассмотрим независимые случайные величины $\pi_{1}(\rho), \ldots, \pi_{s-1}(\rho)$, распределенные по закону Пуассона с параметром $\rho$, случайные величины

$$
l_{r}(\rho)=\sum_{k=1}^{s-1}(s-k) I\left\{\pi_{k}(\rho)=r\right\}, \quad r=1,2, \ldots, R,
$$

и вектор $L_{R}(\rho)=\left(l_{1}(\rho), l_{2}(\rho), \ldots, l_{R}(\rho)\right)$. Распределению вектора $L_{R}(\rho)$ отвечает производящая функция

$$
\varphi\left(z_{1}, \ldots, z_{R}\right)=\prod_{m=1}^{s-1}\left(1+\sum_{r=1}^{R}\left(\frac{\rho^{r}}{r !} e^{-\rho}\left(z_{r}^{m}-1\right)\right)\right) .
$$

Через $\mathcal{L}(\mathcal{Z})$ условимся обозначать распределение случайной величины $Z$. Расстояние по вариации между распределениями случайных величин $\xi$ 
и $\zeta$ обозначим через $d(\mathcal{L}(\xi), \mathcal{L}(\zeta))$. Напомним, что для распределений на множестве неотрицательных целых чисел

$$
d(\mathcal{L}(\xi), \mathcal{L}(\zeta))=\frac{1}{2} \sum_{k=0}^{\infty}|\mathbf{P}\{\xi=k\}-\mathbf{P}\{\zeta=k\}| .
$$

Теорема 1. Пусть натуральные числа $s \geq 2, R \geq 1$. Тогда

$$
d\left(\mathcal{L}\left(M_{R}\right), \mathcal{L}\left(L_{R}\left(n P_{2}\right)\right)\right) \leq 5 s^{3} n P_{3}+\left(1+\left(1-\frac{R}{n}\right)^{-1}\right) s^{2} P_{2} .
$$

Непосредственно из (4) вытекает следующая предельная теорема.

Следствие 1. Пусть $N, n \rightarrow \infty$ так, что $n P_{2} \rightarrow \rho, 0<\rho<\infty$, а параметры $s \geq 1, R \geq 1$ постоянны. Тогда $\mathcal{L}\left(M_{R}\right) \rightarrow \mathcal{L}\left(L_{R}(\rho)\right)$.

ЗАмечание 1. Ранее в работе [3] было показано, что при $N, n \rightarrow \infty$, $s=$ const $\geq 2$ и таком изменении вероятностей $p_{1}, \ldots, p_{N}$, что $n P_{2} \rightarrow \rho$, $0<\rho<1$, вероятность наличия в последовательности $\mathbf{Y}(n, s)$ эквивалентных цепочек с нетривиальной структурой имеет пределом величину $1-e^{-(s-1) \rho}(1+\rho)^{s-1}$. Эта граница достигается именно на цепочках с простейшей структурой.

Теорема 2. Пусть $N, n, s \rightarrow \infty$ так, что $s \leq n u s^{3} n P_{3} \rightarrow 0$. Тогда $\mathbf{P}\left\{\mu_{1}+\mu_{2}+\ldots+\mu_{R}>0\right\} \rightarrow 0$ при любом $R \geq 1$.

Далее потребуются следующие обозначения. Пусть $S^{(1)}(N, s)$ - множество тех структур цепочек длины $s$ из букв алфавита $\{1, \ldots, N\}$, в которых имеется ровно одно совпадение букв. Для структуры из $S^{1}(N, s)$, при которой совпадают знаки на $i$-м и $j$-м местах, $1 \leq i<j \leq s$, будем использовать обозначение « $(i, j) »$. Пусть $S^{(2)}(N, s)$ - множество тех структур, при которых цепочка имеет более одного совпадения букв. Тривиальную структуру удобно обозначить символом «0». Тогда

$$
S(N, s)=S^{(1)}(N, s) \cup S^{(2)}(N, s) \cup\{0\}
$$

множество всех возможных структур цепочек длины $s$ из букв алфавита $\{1, \ldots, N\}$.

В общем случае структуру будем обозначать символом $\sigma$. Число цепочек в $\mathbf{Y}(n, s)$, имеющих структуру $\sigma$, обозначим $u_{\sigma}$. 
При $r=1,2, \ldots, R$ введем обозначения

$$
\begin{aligned}
& \mu_{r}^{(1)}=\sum_{\sigma \in S^{(1)}(N, s)} I\left\{u_{\sigma}=r\right\}=\sum_{1 \leq i<j \leq s} I\left\{u_{i, j}=r\right\}, \\
& \mu_{r}^{(2)}=\sum_{\sigma \in S^{(2)}(N, s)} I\left\{u_{\sigma}=r\right\}, \quad \mu_{r}^{(0)}=I\left\{u_{0}=r\right\} .
\end{aligned}
$$

Тогда

$$
\mu_{r}=\mu_{r}^{(1)}+\mu_{r}^{(2)}+\mu_{r}^{(0)} .
$$

Из определений следует, что

$$
\mu_{r}^{(1)}=\sum_{k=1}^{s-1} \sum_{m=1}^{s-k} I\left\{u_{m, m+k}=r\right\} .
$$

Определим случайные величины

$$
\begin{gathered}
\eta_{k}=\sum_{i=1}^{n} I\left\{X_{i}=X_{i+k}\right\}, \quad k=1, \ldots, s-1, \\
m_{r}^{(1)}=\sum_{k=1}^{s-1}(s-k) I\left\{\eta_{k}=r\right\}, \quad r=1,2, \ldots, R, \\
m_{r}^{(0)}=I\left\{n-\sum_{k=1}^{s-1}(s-k) \eta_{k}=r\right\}, \quad r=1,2, \ldots, R,
\end{gathered}
$$

векторы $\eta=\left(\eta_{1}, \ldots, \eta_{s-1}\right)$ и $\bar{m}_{R}^{(i)}=\left(m_{1}^{(i)}, \ldots, m_{R}^{(i)}\right), i=0,1$.

Рассмотрим события

$D_{1}=D_{1}(n, s)=\{$ в последовательности $\mathbf{Y}(n, s)$ присутствуют $s$-цепочки из $S^{(2)}(N, s)$,

$D_{2}=D_{2}(n, s)=\left\{\right.$ величины $X_{n}, \ldots, X_{n+s-1}$ различны $\}$.

Лемма 1. Выполняется неравенство

$$
\mathbf{P}\left\{M_{R} \neq \bar{m}_{R}^{(1)}+\bar{m}_{R}^{(0)}\right\} \leq \mathbf{P}\left\{D_{1} \cup D_{2}\right\} .
$$

Доказательство леммы. Нетрудно проверить, что

$$
\begin{gathered}
\overline{D_{1}} \cap \overline{D_{2}} \Longrightarrow \\
\Longrightarrow\left\{u_{(i, j)}=\eta_{j-i}, 1 \leq i<j \leq s-1 ; \quad u_{\sigma}(N, n, s)=0, \sigma \in S^{(2)}(N, s)\right\} \Longrightarrow \\
\Longrightarrow\left\{\mu_{r}^{(1)}=m_{r}^{(1)}, \quad \mu_{r}^{(2)}=0, \quad \mu_{r}^{(0)}=m_{r}^{(0)}, r=1,2, \ldots\right\} .
\end{gathered}
$$


Поэтому

$$
\begin{gathered}
\mathbf{P}\left\{M_{R}=\bar{m}_{R}^{(1)}+\bar{m}_{R}^{(0)}\right\} \geq \\
\geq \mathbf{P}\left\{u_{(i, j)}=\eta_{j-i}, 1 \leq i<j \leq s-1 ; u_{\sigma}(N, n, s)=0, \sigma \in S^{(2)}(N, s)\right\} \geq \\
\geq 1-\mathbf{P}\left\{D_{1} \cup D_{2}\right\} .
\end{gathered}
$$

Из (8) следует (7). Лемма доказана.

Лемма 2. Выполняется неравенство

$$
\mathbf{P}\left\{M_{R} \neq \bar{m}_{R}^{(1)}\right\} \leq \mathbf{P}\left\{D_{1} \cup D_{2}\right\}+\frac{s^{2} n P_{2}}{2(n-R)} .
$$

Доказательство леммы. Из определений следует, что

$$
\mathbf{E}\left(\sum_{k=1}^{s-1}(s-k) \eta_{k}\right) \leq \frac{s^{2} n P_{2}}{2} .
$$

Поэтому с помощью неравенства Иенсена приходим к оценке

$$
\mathbf{P}\left\{\bar{m}_{r}^{(0)} \neq \overline{0}\right\}=\mathbf{P}\left\{n-\sum_{k=1}^{s-1}(s-k) \eta_{k} \leq R\right\} \leq \frac{s^{2} n P_{2}}{2(n-R)} .
$$

Теперь (9) следует из (7), (10) и неравенства

$$
\mathbf{P}\left\{M_{R} \neq \bar{m}_{R}^{(1)}\right\} \leq \mathbf{P}\left\{M_{R} \neq \bar{m}_{R}^{(1)}+\bar{m}_{R}^{(0)}\right\}+\mathbf{P}\left\{\bar{m}_{r}^{(0)} \neq \overline{0}\right\} .
$$

Лемма доказана.

Лемма 3. Пусть $n \geq s \geq 3$. Тогда выполнено неравенство

$$
\mathbf{P}\left\{D_{1} \cup D_{2}\right\} \leq \frac{1}{2}(s-1)(s-2)^{2} n\left(P_{3}+P_{2}^{2}\right)+\frac{1}{2}(s-1)_{2} P_{2} .
$$

Лемма 3 является прямым следствием леммы 1 работы [3].

Лемма 4. Для расстояния по вариации между распределениями случайных векторов $\eta=\left(\eta_{1}, \ldots, \eta_{s-1}\right)$ u $\pi\left(n P_{2}\right)=\left(\pi_{1}\left(n P_{2}\right), \ldots, \pi_{s-1}\left(n P_{2}\right)\right)$ выполнено неравенство

$$
d\left(\eta, \pi\left(n P_{2}\right)\right)<2(s-1)^{2} n\left(P_{3}+3 P_{2}^{2}\right) .
$$

Лемма 4 была доказана в работе [3]. 
ЗАМЕчАНИЕ 2. Оценки лемм 3 и 4 внешне несколько отличаются от аналогичных оценок работы [3]. Это объясняется тем, что в [3] рассматривается последовательность $\mathbf{X}$ длины $n$, содержащая $n-s+1$ цепочек длины $s$, а у нас таких цепочек ровно $n$.

Доказательство теоремы 1. Воспользуемся неравенством

$$
d\left(\mathcal{L}\left(M_{R}\right), \mathcal{L}\left(\bar{m}_{R}^{(1)}\right)\right) \leq \mathbf{P}\left\{M_{R} \neq \bar{m}_{R}^{(1)}\right\}
$$

и неравенством

$$
d\left(\mathcal{L}\left(L_{R}\right)\left(n P_{2}\right), \mathcal{L}\left(\bar{m}_{R}^{(1)}\right)\right) \leq d\left(\eta, \pi\left(n P_{2}\right)\right),
$$

вытекающим из идентичности функциональных связей векторов $L_{R}\left(n P_{2}\right)$ и $\bar{m}_{R}^{(1)}$ с векторами $\pi\left(n P_{2}\right)$ и $\eta$ соответственно (см. формулы (2) и (5)). Из (9), (13) и (14) получаем неравенство

$$
\begin{gathered}
d\left(\mathcal{L}\left(M_{R}\right), \mathcal{L}\left(L_{R}\right)\left(n P_{2}\right)\right) \leq \\
\leq \mathbf{P}\left\{D_{1} \cup D_{2}\right\}+\frac{s^{2} n P_{2}}{2(n-R)}+d\left(\eta, \pi\left(n P_{2}\right)\right),
\end{gathered}
$$

подставив в которое оценки (11) и (12), после небольших огрублений получаем (4). Теорема 1 доказана.

Доказательство теоремы 2. Из условий $s \leq n, s^{3} n P_{3} \rightarrow 0$ и неравенства $P_{2}^{2} \leq P_{3}$ следует соотношение $s^{2} P_{2} \rightarrow 0$. Поэтому из (4) и условия $R=$ const следует, что

$$
d\left(\mathcal{L}\left(M_{R}\right), \mathcal{L}\left(L_{R}\left(n P_{2}\right)\right)\right) \rightarrow 0 .
$$

Осталось изучить поведение распределения случайной величины

$$
\mu_{1, R}=\mu_{1}+\mu_{2}+\ldots+\mu_{R}
$$

при $s \rightarrow \infty$. Из формулы (3) следует, что производящая функция распределения этой величины имеет вид

$$
\varphi(z)=\prod_{m=1}^{s-1}\left(1-\Pi_{1, r}\left(n P_{2}\right)+\Pi_{1, r}\left(n P_{2}\right) z^{m}\right),
$$

где

$$
\Pi_{1, R}(\rho)=\sum_{r=1}^{R} \frac{\rho^{r}}{r !} e^{-\rho} \in(0,1) .
$$


Производящая функция $\varphi(z)$ отвечает величине

$$
S_{1, R}=\kappa_{1}+2 \kappa_{2}+\ldots+(s-1) \kappa_{s-1},
$$

где $\kappa_{1}, \kappa_{2}, \ldots, \kappa_{s-1}$ независимые одинаково распределенные индикаторы,

$$
\mathbf{P}\left\{\kappa_{i}=0\right\}=1-\Pi_{1, R}\left(n P_{2}\right), \quad \mathbf{P}\left\{\kappa_{i}=1\right\}=\Pi_{1, R}\left(n P_{2}\right), \quad i=1, \ldots, s-1 .
$$

Поэтому для любого $k=0,1, \ldots, s-2$

$$
\begin{aligned}
& \mathbf{P}\left\{1 \leq \mu_{1, R} \leq k\right\}=\mathbf{P}\left\{1 \leq S_{1, R} \leq k\right\} \leq \\
& \quad \leq \sum_{i=1}^{k} \mathbf{P}\left\{\kappa_{i}=1\right\} \prod_{j=k+1}^{s-1} \mathbf{P}\left\{\kappa_{j}=0\right\}= \\
& =k \Pi_{1, R}\left(n P_{2}\right)\left(1-\Pi_{1, R}\left(n P_{2}\right)\right)^{s-k-1} .
\end{aligned}
$$

При $s \rightarrow \infty$ выражение в правой части этой цепочки соотношений для любого $k$ стремится к нулю независимо от поведения параметра $n P_{2}$. Теорема 2 доказана.

\section{3. Случай, когда среднее число $s$-цепочек \\ с тривиальной структурой ограничено}

Рассмотрим независимые случайные величины $\xi$ и $\eta$, имеющие сложные пуассоновские распределения с производящими функциями

$$
\begin{gathered}
\mathbf{E} z^{\xi}=\exp \left\{\frac{\lambda_{1}(z-1)}{1-(1-\alpha)^{2} z}\right\}, \quad \mathbf{E} z^{\eta}=\exp \left\{\frac{\alpha \lambda(z-1)}{1-(1-\alpha) z}\right\}, \\
\lambda_{1}=\frac{\alpha(2-\alpha) \lambda^{2}}{2}\left(e^{\frac{\alpha^{2}}{2(1-\alpha)^{2}}}-1\right) .
\end{gathered}
$$

Теорема 3. Пусть вероятности $p_{1}=\ldots=p_{N}=1 / N$, а параметры $N, n, s \rightarrow \infty$ таким образом, что

$$
\begin{gathered}
s N^{-1} \rightarrow \alpha, \quad 0<\alpha<1, \\
n(N)_{s} N^{-s} \rightarrow \lambda, \quad 0<\lambda<\infty .
\end{gathered}
$$

Тогда

$$
\begin{aligned}
\mathcal{L}\left(\left(\mu_{2}, \mu_{3}, \mu_{4}, \ldots\right)\right) & \rightarrow \mathcal{L}((\xi+I\{\eta=2\}, I\{\eta=3\}, I\{\eta=4\}, \ldots)) \\
\mathcal{L}\left(\left(n-\mu, n-\mu_{1}\right)\right) & \rightarrow \mathcal{L}((\xi+(\eta-1) I\{\eta \geq 2\}, 2 \xi+\eta I\{\eta \geq 2\})),
\end{aligned}
$$


причем совместное распределение векторов, указанных в левых частях (23) и (24), сходится к совместному распределению векторов, указанных в правых частях этих соотношений.

Предельное распределение рассматриваемого в (23) бесконечномерного вектора является вырожденным. Оно задается сложными пуассоновскими распределениями независимых случайных величин $\xi$ и $\eta$.

Доказательство теоремы 3. Рассмотрим случайные величины

$$
\xi_{r}=\sum_{1 \leq i_{1}<\ldots<i_{r} \leq n} I\left\{Y_{i_{1}}(s) \sim Y_{i_{2}}(s) \sim \ldots \sim Y_{i_{r}}(s)\right\},
$$

равные числу $r$-кратных совпадений структур цепочек в последовательности $\mathbf{Y}(n, s)$. Здесь $r \geq 2-$ натуральное число. Свойства распределений этих случайных величин изучались в работах $[1,2,4-7]$.

Для чисел множеств попарно эквивалентных цепочек в $\mathbf{Y}(n, s)$, состоящих из цепочек с тривиальной и нетривиальной структурой, будем использовать обозначения $\mu_{r}^{(0)}$ и $\mu_{r}^{(1,2)}=\mu_{r}^{(1)}+\mu_{r}^{(2)}$ соответственно. Введем также случайные величины:

$\xi_{r}^{(1,2)}$ - число наборов по $r$ цепочек с одинаковой нетривиальной структурой в последовательности $\mathbf{Y}(n, s)$,

$\xi_{r}^{(0)}-$ число наборов по $r$ цепочек с тривиальной структурой в последовательности $\mathbf{Y}(n, s)$,

$\zeta$ - число цепочек с тривиальной структурой в $\mathbf{Y}(n, s)$.

Ясно, что

$$
\xi_{r}=\xi_{r}^{(1,2)}+\xi_{r}^{(0)}, \quad \xi_{r}^{(0)}=C_{\zeta}^{r}, \quad r \geq 2
$$

Тогда

$$
\begin{gathered}
\xi_{r}^{(0)}=C_{\zeta}^{r}, \quad \mu_{r}^{(0)}=I\{\zeta=r\}, \quad \mu_{r}=\mu_{r}^{(1,2)}+I\{\zeta=r\}, \\
\xi_{r}=\xi_{r}^{(1,2)}+C_{\zeta}^{r}, \quad \xi_{r}^{(1,2)}=\sum_{k=r}^{n} C_{k}^{r} \mu_{r}^{(1,2)} .
\end{gathered}
$$

Свойства случайных величин $\xi_{r}^{(1,2)}$ и $\zeta$ исследовались в работе [7]. Воспользуемся одной из теорем этой работы, которая в нашем случае может быть сформулирована следующим образом. 
Теорема А. Пусть выполнены условия теоремы 3. Тогда $\mathcal{L}\left(\left(\xi_{2}^{(1,2)}, \zeta\right)\right) \rightarrow \mathcal{L}((\xi, \eta))$ и $\xi_{r}^{(1,2)} \rightarrow 0$ по вероятности, $r \geq 3$, где случайные величины и п те же, что в теореме 3.

Из теоремы А, соотношений (26), (27) и

$$
\mu_{1}+2 \mu_{2}+\ldots+n \mu_{n}=n, \quad \mu=\mu_{1}+\mu_{2}+\ldots+\mu_{n}
$$

следует заключение теоремы. Теорема 3 доказана.

Авторы признательны А. М. Зубкову за полезные замечания.

\section{Список литературы}

1. Буравлев С.М. Повторения с точностью до перестановок в последовательности независимых испытаний // Дискретная математика. - 1999. T. 11. Вып. 2. - С. 53-75.

2. Михайлов В.Г., Шойтов А.М. Структурная эквивалентность $s$-цепочек в случайных дискретных последовательностях // Дискретная математика. - 2003. - Т. 15. Вып. 4. - С. 7-34.

3. Михайлов В.Г. Об асимптотическом поведении вероятности наличия в последовательности эквивалентных цепочек с нетривиальной структурой // Дискретная математика. - 2008. - Т. 20. Вып. 4. C. $113-119$.

4. Михайлов В.Г. Об асимптотических свойствах распределения числа пар $H$-связанных цепочек // Дискретная математика. - 2002. - Т. 14. Вып. 3. - С. 122-129.

5. Шойтов А. М. Пуассоновское приближение для числа повторений значений дискретной функции от цепочек // Дискретная математика. - 2005. Т. 17. Вып. 2. - С. 56-69.

6. Шойтов А. М. Дискретные предельные распределения для числа цепочек с одинаковой структурой в последовательности равновероятных испытаний // Обозрение прикладной и промышленной математики. - 2007. Т. 14. Вып. 3. - С. 474-484.

7. Шойтов А.М. Структурно эквивалентные цепочки в равновероятной полиномиальной схеме // Математические вопросы криптографии. 2012. - Т. 3. Вып.3. - С. 129-151. 